The following pages constitute the final, accepted and revised manuscript of the article:

$$
\text { Merlo, Juan }
$$

"Changing analytical approaches in European epidemiology - a short comment on a recent article."

Eur J Epidemiol. 2005;20(8):737.

Publisher: Springer.

Use of alternative location to go to the published version of the article requires journal subscription.

Alternative location: http://dx.doi.org/10.1007/s10654-005-2065-9 


\title{
Changing analytical approaches in European epidemiology - a short comment on a recent article
}

\author{
European Journal of Epidemiology (2005) 20: 737 _ Springer 2005 \\ DOI 10.1007/s10654-005-2065-9 \\ Juan Merlo \\ Department of Clinical Sciences, Faculty of Medicine, Lund University, Malmo“, Sweden \\ Address for correspondence: \\ Juan Merlo, Associate Professor in Public Health Medicine \\ Department of Clinical Sciences, Faculty of Medicine, Lund University, Malmö \\ Sweden \\ Phone: +040-332064 \\ E-mail: Juan.merlo@med.lu.se
}

Multilevel analysis is today considered a more appropriate way to monitor health care performance, as it allows a less biased estimation of uncertainty, and can also separate and quantify contextual (as opposed to individual) effects. Multilevel analysis should be applied as the standard methodology for hospital comparisons. A greater awareness of this question in future Journal articles may be in order.

Key words: Hospital comparisons, Multilevel analysis

My colleagues and I have an article in press that will shortly appear in the journal Medical Care [1]. It explores an issue indirectly related to a recently published study in the European Journal of Epidemiology by Capon et al. [2]. We investigate variance in neonatal mortality in Sweden, while the study by Capon et al. is concerned with variability in caesarean section in Italy. The data in both articles are based on a large number of hospitals and deliveries, and both have a clear methodological style. In fact, the title of Capon's article itself suggests a methodological concern.

I was attracted by the question raised by Capon et al. and read their paper with considerable interest. It was rather disappointing to find that this article, although it presents itself as methodologically founded, avails itself of a rather old-fashion epidemiology. In doing so, it naively overlooks the modern methodological considerations pertaining to hospital comparisons.

The authors begin with a clear exposition of the hierarchical structure of their data, outlining factors related to the mother, physician profiles, and hospital characteristics as elements that may contribute to the propensity for caesarean sections. However, they do not at all mention that this multilevel structure conditions a dependence on the residuals that needs be dealt with by employing suitable analytical techniques. The authors include in their model 60 dummy variables identifying single hospitals, instead of a simple random term for hospital effects. This outmoded procedure contains many disadvantages and has been seriously criticised for several years [3, 4], especially since appropriate epidemiological techniques, such as multilevel regression analysis, have been well-developed in Europe [5].

Multilevel analysis is today considered a more appropriate way to monitor health care performance, as it allows a less biased estimation of uncertainty, and can also separate and quantify contextual (as opposed to individual) effects. Multilevel analysis should be applied as 
the standard methodology for hospital comparisons. A greater awareness of this question in future Journal articles may be in order.

\section{References}

1. Merlo J, Gerdtham U-G, Eckerlund I, et al. Hospital level of care and neonatal mortality in low and high risk deliveries - reassessing the question in Sweden by Multilevel Analysis. Med Care 2005, (In press).

2. Capon A, Di Lallo D, Perucci CA, Panepuccia L. Casemix adjusted as an alternative way to compare hospital performances odds ratios. Eur J Epidemiol 2005; 20: 497-500.

3. Goldstein H, Spiegelhalter D. League tables and their limitations: Statistical issues in comparisons of institutional performance. J Roy Stat Soc Ser A 1996; 159: 385-443.

4. Leyland AH, Boddy FA. League tables and acute myocardial infarction. Lancet 1998; 351: 555-558. 5. Rasbash J, Steele F, Brown W. A User's Guide to MLwiN. Version 2.0. Documentation Version 2.1e. Centre for Multilevel Modelling, Institute of Education. London: University of London, 2003. 